\title{
Fallopian Tube Prolapse after Abdominal Hysterectomy: A Rare Cause of Chronic Pelvic Pain
}

Mariana Ormonde ${ }^{1}$, Maria Inês Raposo1, Mariana Cardoso', Andrea Pereira ${ }^{1}$, Rui Mendonça ${ }^{1}$, Carlos Ponte ${ }^{1}$ 1- Serviço de Ginecologia e Obstetrícia do Hospital do Divino Espírito Santo de Ponta Delgada, EPE

Problem statement

Hysterectomy is the most common major surgery performed in Gynecology. A rare complication of this procedure is fallopian tube prolapse, which is thought to occur in only $0,1 \%$ of all hysterectomies, more often after vaginal hysterectomy than laparotomy. The most frequent symptoms are: profuse watery or bloody vaginal discharge, chronic pelvic pain and deep dispareunia. Because of its rareness and insufficient knowledge about this condition, fallopian tube prolapse is usually misdiagnosed as granulation tissue. Therefore, delayed treatment is frequent, which prolongs patient's discomfort. A biopsy of the suspected prolapsed tube may be performed, because final diagnosis is carried out by histopathologic characterization. Still, some surgeons decide to perform surgery without previous biopsy, when prolapsed fallopian tube is strongly suspected. Definite treatment is achieved by total salpingectomy, which can be performed by vaginal approach, laparotomy or laparoscopy, depending on various factors, such as surgeon's skill. After surgical treatment, the majority of patients become asymptomatic and typically no recurrence is reported.

\section{Methods}

Retrospective study of a clinical case from our center and review of the literature.

\section{Clinical Case}

A 34 year-old multiparous woman, smoker, was referred to our center for Low-grade Squamous Intraepithelial Lesion (LSIL), in 2011. After satisfactory colposcopy, guided biopsy was performed, which revealed Cervical Intraepithelial Neoplasia (CIN) 3. She underwent conization two times (2011 and 2014) for recurrence of the disease. In 2016, because of lesion persistence, an abdominal hysterectomy was performed. Surgery went as expected and postoperative recovery was uneventful. Patient was discharged after $48 \mathrm{~h}$, with no immediate complications.

Multiple visits to the Emergency Department

during 6 months

\section{Severe Pelvic Pain}

"Urine Incontinence"

X Normal laboratory work-up;

$X$ Normal Pelvic Ultrasound;

$X$ No vesico-vaginal fistula found by the Urologist;

$X$ Profuse watery and blood discharge, foul-smelling;

$X$ Polypoidal, strawberry colored growth $(2 \times 2 \mathrm{~cm})$, pending from the vaginal cuff;

$X$ Pelvic pain reproduced by traction of the polypoidal growth and vaginal palpation.

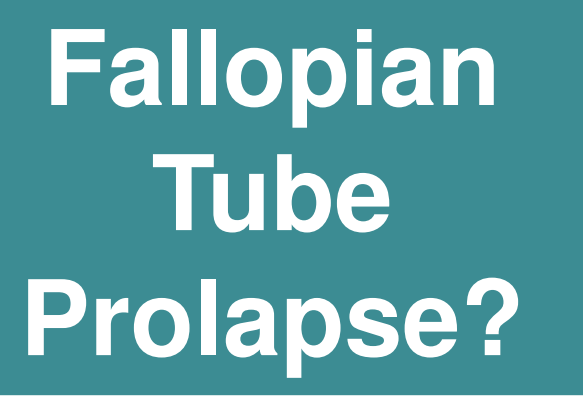

Exploratory Laparotomy

Bilateral Salpingectomy and vaginal cuff closure

Figure 1. Findings during physical examination and surgery. (A) Fallopian tube fimbriae and watery discharge (arrow); (B) Right fallopian tube "imprisoned" in the vaginal cuff (arrow). (C) Right fallopian tube after salpingectomy, with edematous and necrotic appearance.
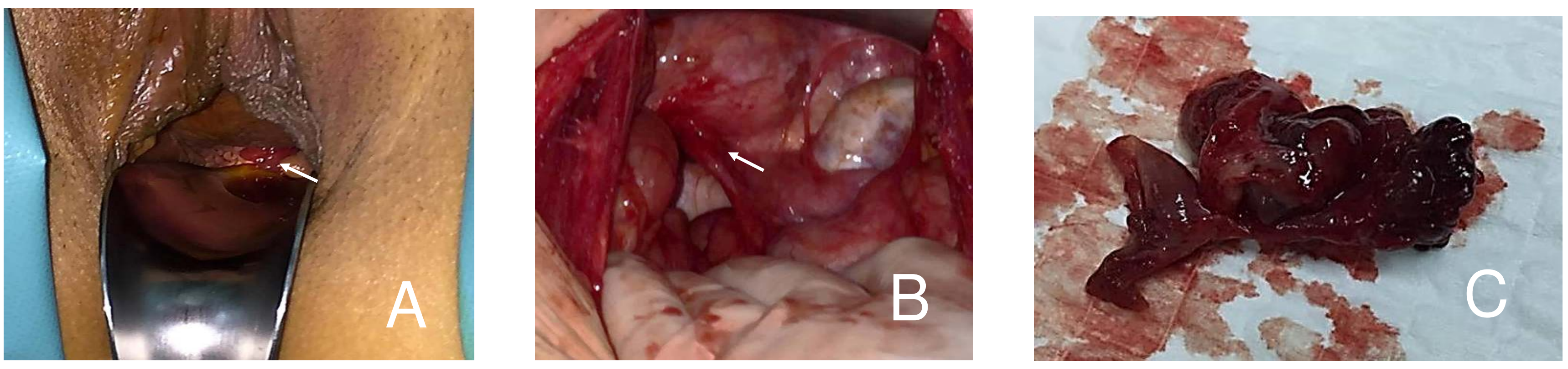

X Histopathological examination: usual fallopian tube histology, with chronic inflammation and necrotic foci;

$\mathrm{X}$ At follow-up, patient was asymptomatic and the vaginal cuff was scarred.

\section{Conclusion}

Fallopian tube prolapse after abdominal hysterectomy is uncommon but gynecologists should be aware of this possible complication because it affects greatly women's quality of life, including daily and sexual life. Definitive treatment is achieved by total salpingectomy, and the approach (vaginal, laparotomy or laparoscopy) should be decided case-by-case and depending on the surgeon's experience. After surgical treatment, the majority of patients become asymptomatic and usually no recurrence is reported. 